\title{
Alpha gal specific IgE positivity due to tick bites and red meat allergy: the first case report in Turkey
}

\author{
Şennur Keleş ${ }^{1}$, Mehmet Gündüz ${ }^{2}$ \\ ${ }^{1}$ Pediatric Allergy and Immunology, University of Health Sciences Antalya Training and Research Hospital, \\ Antalya; ${ }^{2}$ Department of Pediatrics, Division of Neonatology, Bağcllar Medipol Mega University Hospital, Istanbul. \\ E-mail: sennurkeles@hotmail.com \\ Received: 10th November 2017, Revised: 19th July 2018, 30th July 2018, Accepted: 14th August 2018
}

SUMMARY: Keleş Ş, Gündüz M. Alpha gal specific IgE positivity due to tick bites and red meat allergy: the first case report in Turkey. Turk J Pediatr 2019; 61: 615-617.

Galactose-alpha-1,3-galactose is a carbohydrate moiety found in the cell membranes of many organisms, commonly known as alpha-gal $(\alpha-\mathrm{Gal})$. This oligosaccharide is not found in the group of primates in which humans are involved. It has been found that $\alpha-G a l$ plays a key role in delayed IgE-mediated red meat allergy and this allergen is triggered by tick bites. Here we present a 7-year-old boy with alpha-gal allergy from Turkey.

Key words: alpha- gal allergy, child, delayed urticaria and anaphylaxis, meat allergy, tick bite.

Delayed anaphylaxis, urticaria or angioedema to red meat is an allergy that has been increasingly recognized over the last few years. The mechanism involves an IgE reaction to an oligosaccharide, galactose-alpha-1,3-galactose (alpha-gal), found in non-primate mammals and tick glycoproteins. Over the course of evolution, primates and humans have lost enzyme required for alfa-gal production. As a result this disaccharide is immunogenic in humans. The reaction can consist of anaphylaxis, urticaria or angioedema and occurs in response to ingestion of mammalian meat, such as beef, lamb, pork, or venison. The sensitization most likely occurs by induction of IgE through tick bites, particularly to Amblyomma americanum in the U.S., but also to Ixodes species in other countries, such as Australia, Europe, Central America, and Asia. $^{1-4}$

This is a first case report in Turkey who sustained tick bites and subsequently developed an allergic reaction to red meat.

\section{Case Report}

Our patient is a 7-year-old male child. His family reported that for the last two months, he had had episodes of diffuse hives intermittently and two weeks prior to the admission, there were hives and co-morbid nausea. His complaints resolved after taking antihistamines at home. He had no personal or family history of atopy. His physical examination was normal. The percentage of eosinophil (9.8\%), total IgE $(326 \mathrm{IU} / \mathrm{ml})$, other Ig $\mathrm{G}$, Ig A, Ig $\mathrm{M}$ and biochemical parameters, urine and stool examinations were normal. Skin prick tests performed with commercial extracts for a standard panel of food and inhalant allergens, resulted as negative. His blood type was A positive. When the story was questioned in detail, family reported that the hives might have been due to consumption of red meat, and he had been bitten by ticks twice a year ago. Red meat elimination was carried out for three weeks and no hives developed during this period. Skin prick tests, performed with using fresh meat; beef (9-9 
$\mathrm{mm}$ in diameter) and mutton (10-11 $\mathrm{mm}$ in diameter) were positive. In the fifth hour of the food provocation test with beef, urticarial plaques were observed. His $\alpha$-Gal specific IgE (galactose-alpha-1,3-galactose IgE) level was $2.87 \mathrm{kU} / \mathrm{L}$, beef specific IgE level was 0.1 $\mathrm{kU} / \mathrm{L}$. He has remained symptom-free while avoiding red meat. Adrenaline auto-injector was prescribed for emergency use.

Informed consent was obtained from the patient's family.

\section{Discussion}

IgE to alpha-gal has been associated with two distinct forms of anaphylaxis; 1) immediate onset anaphylaxis during first exposure to intravenous cetuximab, and 2) delayed onset anaphylaxis 3-6 hours after red meat ingestion. ${ }^{3}$

An IgE antibody response to alpha-gal was first recognized when patients treated with monoclonal antibody cetuximab experienced anaphylactic reactions upon the first injection. ${ }^{4}$ Investigations established that the reactions to cetuximab occurred in patients who had preexisting IgE antibodies to the oligosaccharide on the Fab portion of this molecule. Patients who, having been bitten by ticks, have developed these kinds of IgE antibodies. ${ }^{5}$

The proposed mechanism of sensitization is that the tick bite stimulates production of IgE to alpha-gal, and the $\operatorname{IgE}$ then binds to high-affinity IgE receptors on mast cells and basophils. When mammalian meat is later ingested by a sensitized individual, the alpha-gal antigen in the meat binds the IgE, which results in the release of mediators, such as histamine, from these cells. The characteristics of red meat allergy are different from typical allergic reactions. Classic IgE mediated anaphylaxis occurs within one hour of allergen exposure. However, anaphylaxis and urticaria due to IgE antibody to alpha -gal, a carbohydrate, occurs on average three to six hours after ingestion of alfa-gal containing red meat. The delayed anaphylaxis is hypothesized to biochemistry, digestional absorption of alpha-gal containing glycolipids and glycoproteins. ${ }^{6-9}$
Our patient's beef specific IgE was negative and this finding is not consistent with the literature on alpha-gal but in one adult, alphagal sensitized case report who had anaphylaxis to medications containing meat byproducts also had negative beef immunoassay. Other allergic sources of alpha-gal containing drugs, such as gelatins, magnesium stearate are currently rare but have to be taken into account. ${ }^{10}$ Our patient had no history of drug use and we could not explain why beef specific IgE result was negative.

The molecular structure of $\alpha$-Gal is similar to that of the $\mathrm{ABO}$ blood group $\mathrm{B}$ antigen, a self-antigen in patients with blood types $B$ or $\mathrm{AB}$. This molecular similarity provokes the hypothesis that patients who harbor the $\mathrm{B}$ antigen are less likely to undergo allergic sensitization to $\alpha$-Gal and develop red meat allergy. ${ }^{11}$ Our patient's blood type was A positive and supported this hypothesis.

In conclusion, to our knowledge this is the first reported case of red meat allergy due to tick bite in Turkey. If a patient has unknown etiology of delayed type anaphylaxis and urticaria, especially with tick bite history, red meat allergy must be taken into account and referred to an allergy expert.

\section{REFERENCES}

1. Commins SP, Satinover SM, Hosen J, et al. Delayed anaphylaxis, angioedema, or urticaria after consumption of red meat in patients with $\operatorname{IgE}$ antibodies specific for galactose- $\alpha$-1,3-galactose. J Allergy Clin Immunol 2009; 123: 426-433.

2. Commins SP, Platts-Mills TAE. Tick bites and red meat allergy. Curr Opin Allergy Clin Immunol 2013; 13: 354-359.

3. Steinke JW, Platts-Mills TAE, Commins SP. The alphagal story: Lessons learned from connecting the dots. J Allergy Clin Immunol 2015; 135: 589-596.

4. Commins SP, Platt-Mills TAE. Anaphylaxis syndrome related to a new mammalian cross-reactive carbohydrate determinant. J Allergy Clin Immunol 2009; 124: 652-657.

5. Chung $\mathrm{CH}$, Mirakhur B, Chan E, et al. Cetuximabinduced anaphylaxis and IgE specific for galactosealpha-1,3-galactose. N Engl J Med 2008; 358: 1109 1117.

6. Calamari AM, Poppa M, Villalta D, Pravettoni V. Alpha-gal anaphylaxis: The first case report in Italy. Eur Ann Allergy Clin Immunol 2015; 47: 161-162. 
7. Kaloga M, Kourouma S, Kouassi YI, et al. Allergy to red meat: A diagnosis made by the patient and confirmed by an assay for IgE antibodies specific for alpha-1,3-galactose. Case Rep Dermatol 2016; 8: 10 13.

8. Platts-Mills TAE, Schuyler AJ, Hoyt AEW, Commins SP. Delayed anaphylaxis involving IgE to galactosealpha-1,3-galactose. Curr Allergy Asthma Rep 2015; 15: 512 .

9. Kennedy JL, Stallings AP, Platts-Mills TAE, et al. Galactose-alpha-1,3-galactose and delayed anaphylaxis, angioedema, and urticaria in children. Pediatrics 2013; 131: e1545-e1552.
10. Muglia C, Kar I, Gong M, Hermes-DeSantis ER, Monteleone C. Anaphylaxis to medications containing meat byproducts in an alpha-gal sensitized individual J Allergy Clin Immunol Pract 2015; 3: 796-797.

11. Brestoff JR, Tesfazghi MT, Zaydman MA, et al. The B antigen protects against the development of red meat allergy. J Allergy Clin Immunol Prac 2018; 6: 17901791.e3. 